\title{
A medical study on wireless inertial measurement technology as a tool for identifying patients at risk of death or imminent clinical deterioration
}

\author{
Michael Walsh, Mark Gaffney, John Barton, \\ Brendan O'Flynn, Cian O'Mathuna, \\ Clarity Centre for Web Technologies, \\ Tyndall National Institute, \\ University College Cork, \\ Cork, Ireland
}

\begin{abstract}
This paper provides a system description and preliminary results for an ongoing clinical study currently being carried out at the Mid-Western Regional Hospital, Nenagh, Ireland. The goal of the trial is to determine if wireless inertial measurement technology can be employed to identify elderly patients at risk of death or imminent clinical deterioration. The system measures cumulative movement and provides a score that will help provide a robust early warning to clinical staff of clinical deterioration. In addition the study examines some of the logistical barriers to the adoption of wearable wireless technology in front-line medical care.
\end{abstract}

Keywords- wireless inertial measurement; clinical study

\section{INTRODUCTION}

On admission to hospital the mental and functional status of patients and their risk of dying are currently assessed subjectively by nursing and medical staff. In order to make this process more objective and quantifiable several scoring systems to measure these parameters are in development. These include the RAS mental status score, the Falls Risk Assessment Score and the Simple Clinical Score [1-3]. Potentially many of the measurements on which these scores are based could be performed by non-invasive electronic devices. Introduction of this technology is currently limited mainly because not enough is known about its reliability and robustness in "real life" clinical practice.

Functional status is a major determinant of mortality [2]. The inability to stand and/or walk unaided has been shown to identify acutely ill patients with a greatly increase risk of mortality. Wireless inertial measurement has been used to identify frail patients at increased risk of falls, but its ability to identify patients at risk of death or imminent clinical deterioration has not been examined.

Currently all patients admitted to the Elderly Care Unit in Nenagh Hospital are assessed using multiple scoring systems, and all the parameters of these scores are being entered into a computer database. This score is subsequently used to determine the intensity of care required (see figure 1). This work proposes the attachment of a wireless inertial measurement unit (WIMU) to all these patients for the first 24 hours after their admission. These devices transmit all movement recorded during this time. These continuous streams

\author{
Anne Hickey ${ }^{1}$, John Kellett ${ }^{2}$, \\ ${ }^{1}$ Nurse Manager, ${ }^{2}$ Consultant Physician, \\ ${ }^{1}$ Elderly Care Unit, \\ Mid-Western Regional Hospital, Nenagh, \\ County Tipperary, Ireland
}

of data are subsequently analyzed to determine if different patterns of movement can be correlated with the currently used clinical scores, other clinical findings and subsequent clinical outcomes. A "Movement Score" will subsequently be developed that will help provide a robust early warning to clinical staff of clinical deterioration.

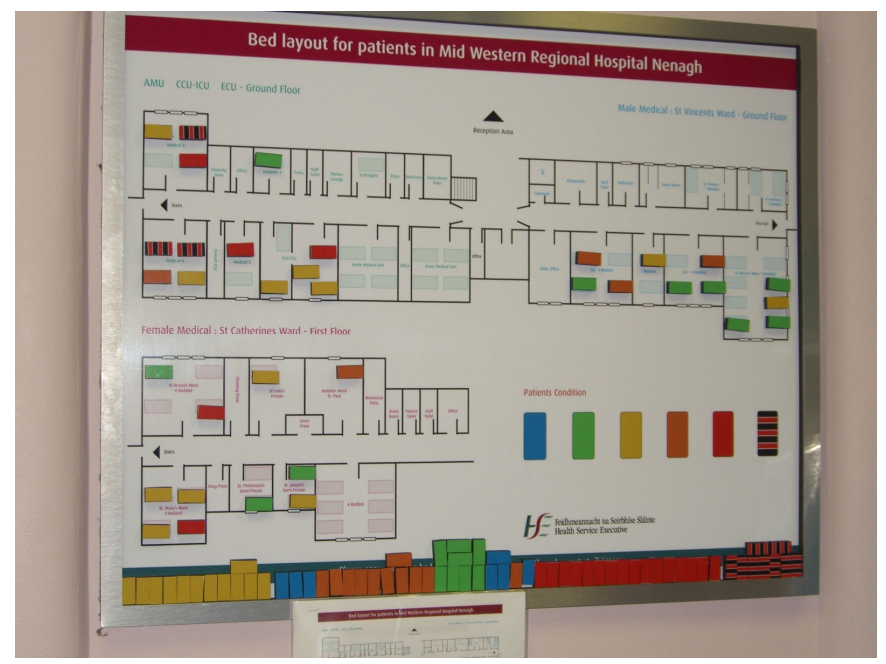

Figure 1. The patient's condition is represented by a colour where blue and black/red strips indicates a low risk and critical subject respectively. This in turn determines the intensity of care required.

\section{HARDWARE DESCRIPTION}

\section{A. Wireless Inertial Measurement Units}

In the first instance WIMUs were designed and manufactured [4], [5]. The WIMU is comprised of two separate modular components. The data processing and communications module consists of the Atmega1281 microcontroller (Atmel Corp.) and the EM2420 (Ember Corp.) 802.15.4 compliant transceiver. The inertial module has onboard circuitry for battery recharging and signal conditioning. It includes the ADXL330 (Analog Devices, Inc) low power, complete 3-axis accelerometer. This component was selected as it can capture dynamic movement as well as static acceleration of gravity in tilt-sensing applications. The WIMU is programmed using the TinyOS embedded operating system. Sampling frequency for this study was set to $200 \mathrm{~Hz}$. Care was given when selecting 


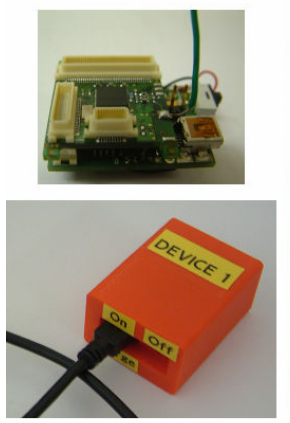

(a)

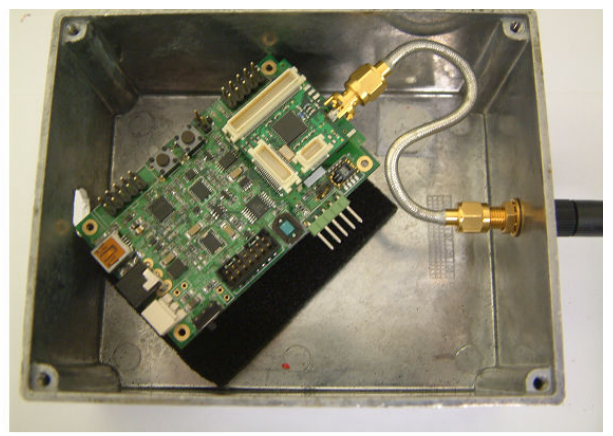

(b)
Figure 2. (a) WIMU Technology (top), Packaged and charging (bottom), (b) Shielded base station unit

communications channel so as to avoid interference from the Wifi. The WIMU forwards time stamped data to the base station unit shown in Figure 2 which in turn sends data via USB to a PC.

1) Tranmission redundancy to overcome lossy wireless links: Wireless networks are inherently lossy due mainly to the propagation environment in which they operate. While most non-safety critical applications will ignore that some data will inevitably be lost this attribute is less acceptable in the clinical environement. To overcome this limitiation a custom multihopping protocol was developed for this work. To illustrate how the protocol works the elderly care unit at Nenagh hospital, where the trial is ongoing, is shown in figure 3 . The base station unit is located in the nurses office. There are two mains powered repeater nodes which relay data from the WIMUs to the base station. Rather then establishing a route to the central access point based on link quality or network traffic as is generally the case in multitier networks, this protocol allows the data travelling through the network to arrive at the base station via multiple paths.

For example WIMU2 in figure 3 sends data via the R1 repeater and also directly to the base station. The base station in turn forwards the information via USB to a personal computer where a Perl script identifies two packets from the same WIMU based on time stamp information and discards the packet with the poorest bit error rate (the data least corrupted by wireless transmission). This simple method in turn negates the requirement for multiple retransmissions from the battery constrained WIMUs while increasing the probability that the safety critical data will be recorded efficiently.

\section{B. Packaging}

Packaging the WIMUs so that they are deployable in the clinical setting is a key element to the adoption of this type of wearable technology. A number of enclosures were designed as illustrated in figure 4 ensuring the devices would be as user friendly as possible while also adhering to the stringent hygiene requirements of hospital use. Figure 5 illustrates the custom made plastic enclosure selected for use in the trial which exposes an on/off switch and a mini USB connection for recharging the device. Following consultation with the nursing staff a disposable outer enclosure made from polyester cloth was designed to ensure the devices remained sanitary and to enable the nurses to attach the devices to the patient so as not to damage the subjects clothing (the nurses reported that this was an important and yet often overlooked aspect of introducing a new device to the patient). Two clips at either end of the cloth enclosure also ensured minimal artifact was present in the recorded signal.

\section{Nenagh Hospital Elderly Care Unit - 11 beds}

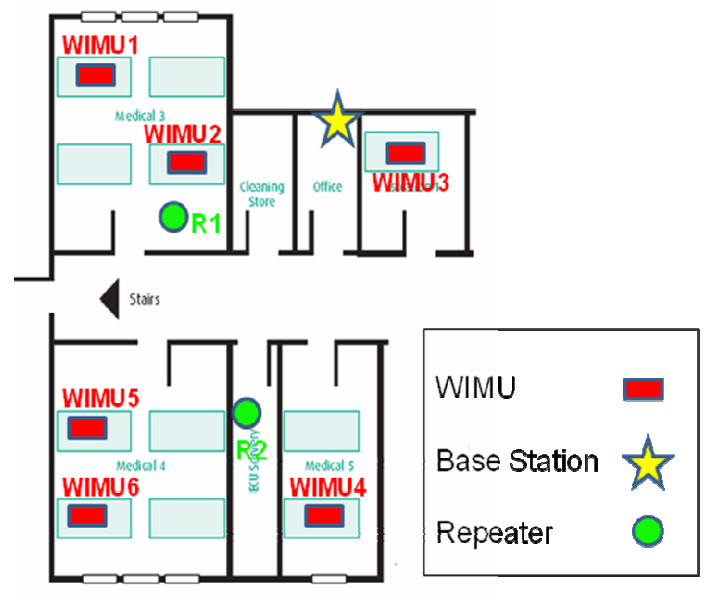

Figure 3. Network topology for the Elderly Care Unit

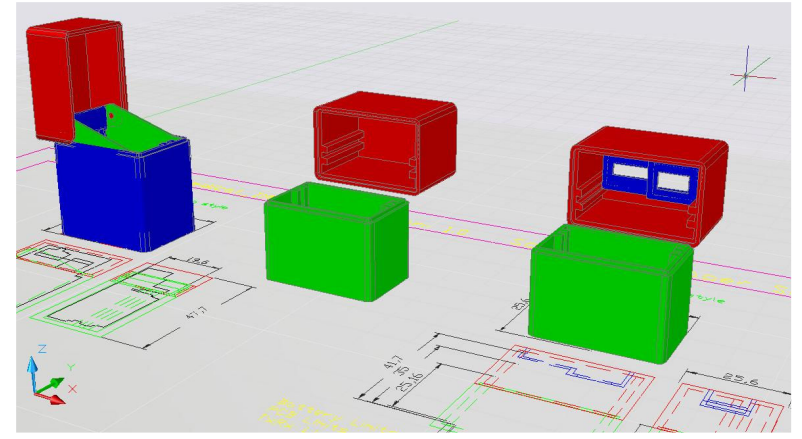

Figure 4. Custom plastic enclosure design for WIMU

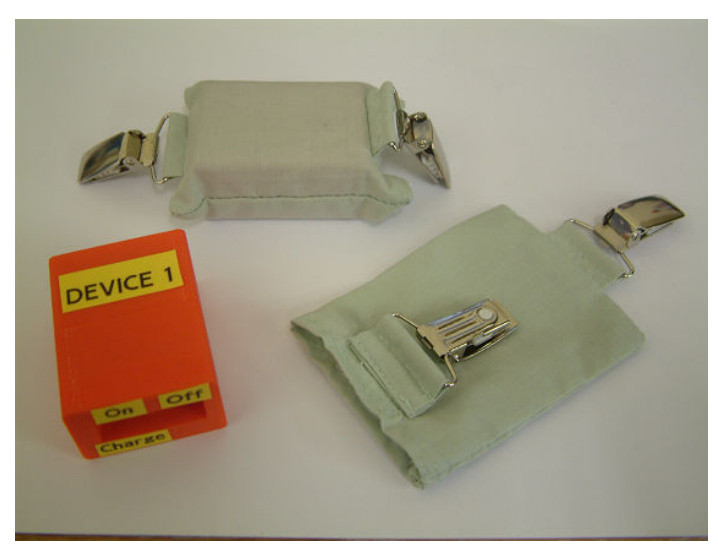

Figure 5. Cloth enclosure for WIMU ensuring the devices remain sterile while minmising artifact introduction. 


\section{MOVEMENT SCORE AND FIRMWARE DESCRIPTION}

\section{A. Calculating a "Movement Score"}

As mentioned above a Perl script captures data forwarded over USB and parses the information. The script logs raw accelerometer data to file and calculates movement $\sigma$ on a per sample basis as follows:

$$
\sigma=\sum_{k=2} \sqrt{\left(x_{k}-x_{k-2}\right)^{2}+\left(y_{b}-y_{k-2}\right)^{2}+\left(y_{k}-y_{k-2}\right)^{2}}
$$

where $k$ is the sample number, $n$ is the number of samples per minute, $x_{k}, y_{k}$ and $z_{k}$ are the $\mathrm{x}$-axis, $\mathrm{y}$-axis and $\mathrm{z}$-axis accelerometer readings at sample $k$. This simple approach measures cumulative movement overtime including all three axes. As it is singularly the variation between subsequent samples that is measured this makes the score independent of acceleration due to gravity and more robust to sensor drift over prolonged periods of measurement. A "Movement Score" ranging between -5 and 5 is arrived at using empirical data gathered from a derivation cohort of patients.

\section{B. Labview Front End Graphical User Interface}

A user friendly GUI has been developed as part of this work and is shown in figure 6 . The GUI is designed with nursing needs in mind and following further consultation with the nursing staff the following functionalities were included:

- The 'Add Patient' button shown in figure 7(a) allows a patient to be added to the system. Once pressed the nurse is required to input patient name, patient hospital number and the number of the WIMU to be attached (printed on the device see figure 5).

- The 'Remove Patient' button allows a patient to be removed from the system following 24 hours of assessment (figure 7(b)).

- If an event of importance occurs while the patient is instrumented an 'Add Note' button allows the nurse to input details of the event. For instance if a patient were to have a fall, become unresponsive or exhibit agitation this would be recorded. These events are later correlated with WIMU data to determine if the event has been flagged by the system.

- The nurse is able to view how the Movement Score varies overtime for an instrumented patient by pressing the 'View Trend' button as illustrated by figure 8 .

- A 'Device Active' LED light indicates that the device is functioning as it should. If the device is switched off, running low on battery or out of wireless communications range this light switches off.

- An 'Activity Score' correlation tab is provided to inform the observer of how to interpret the Movement Score. Here a score of 0 indicates the patient exhibits normal activity, a figure of 5 indicates the patient exhibits excessive activity and a score of -5 informs the observer that the patient has not moved at all.

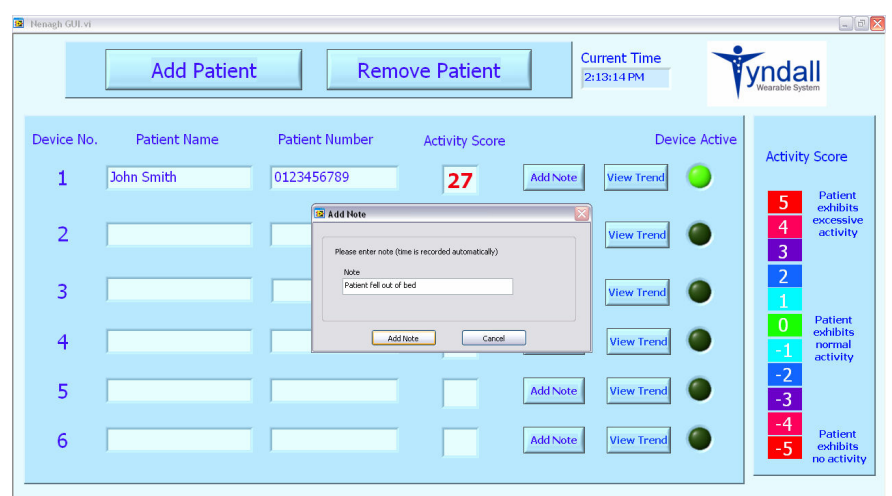

Figure 6. Labvire user friendly based GUI

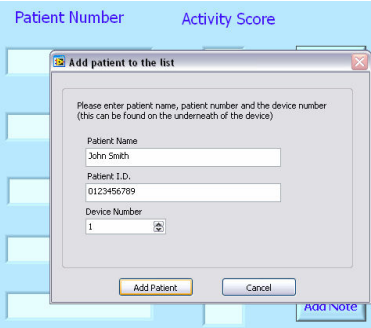

(a)

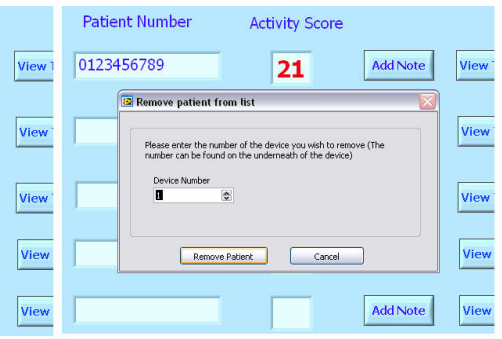

(b)
Figure 7. (a) Adding a patient to the system, (b) Removing a patient from the system.

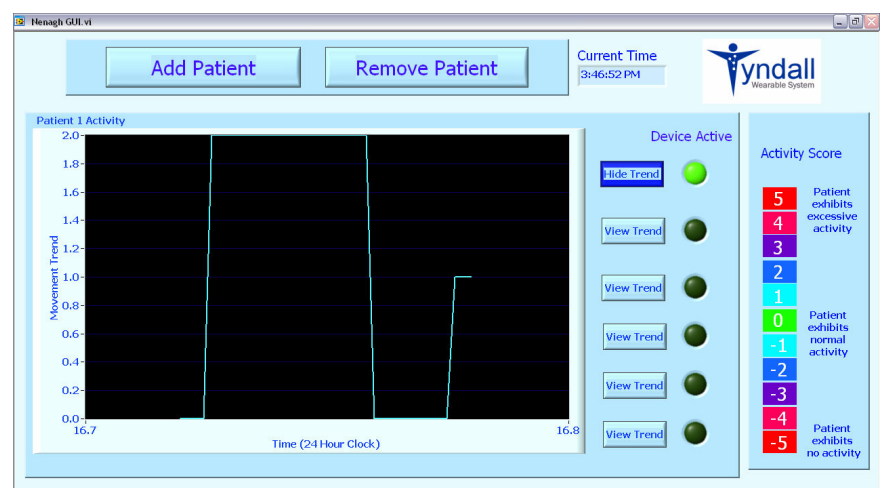

Figure 8. Viewing the "Movement Score" trend overtime

\section{PRELIMINARY RESULTS, DisCUSSION AND FUTURE WORK}

As mentioned previously upon admission to the MidWestern Regional Hospital in Nenagh currently all patients are assessed using multiple scoring systems and all the parameters of these scores are entered into a computer database. The medical assessment result that is output by this assessment is illustrated in figure 9 and requires as inputs demographic details, co-morbid condition, presenting complaint, mental and functional status and a number of physiological measurements including electrocardiogram, temperature, blood pressure, pulse and respiration rates and blood oxygen saturation. Estimated risk of falls, risk of a patient developing a pressure sore, length of stay, risk of mortality (within 24 hours and 30 days), life expectancy and intensity of care required are calculated based on these inputs. 

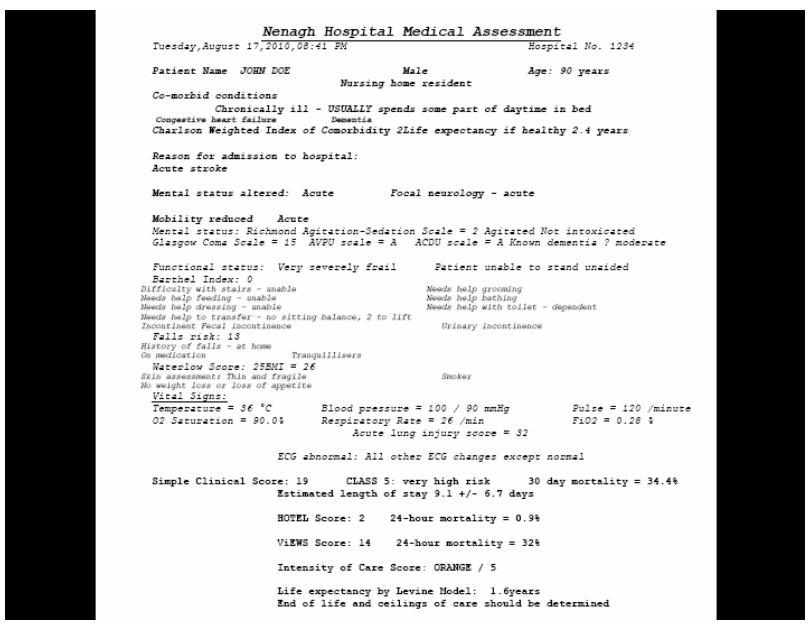

Figure 9. Mid-Western Regional Hospital, Nenagh Medical Assessment

For the purpose of this work this assessment is carried out twice once upon admission and then again following 24 hours of the patient being instrumented with a WIMU. To date data from 20 patients has been gathered which will form $50 \%$ of the derivation cohort of 40 patients required to arrive at a Movement Score curve fitting database. Raw WIMU data gathered over an 8 hour period is shown in figure 10 for a patient noted by the nursing staff as having exhibited agitated behavior at recorded times. Figure 11 highlights how the Movement Scoring methodology can highlight these periods of excessive patient activity.

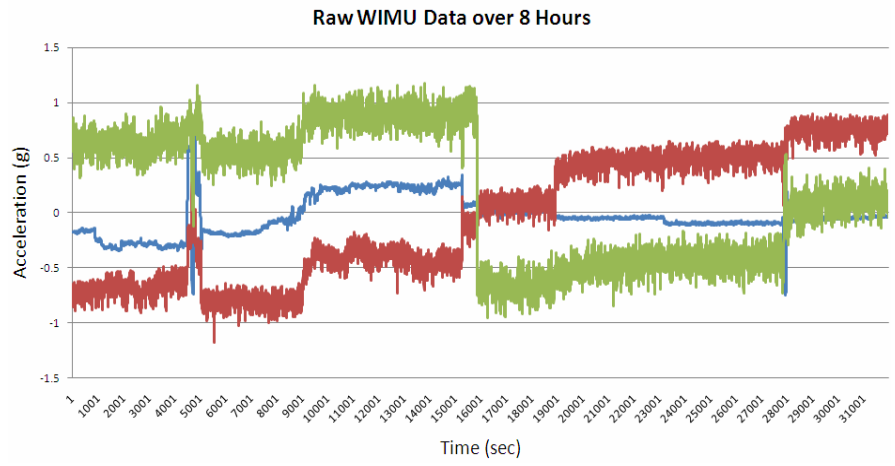

Figure 10. Raw WIMU data gathered over 8 hours from a single patient

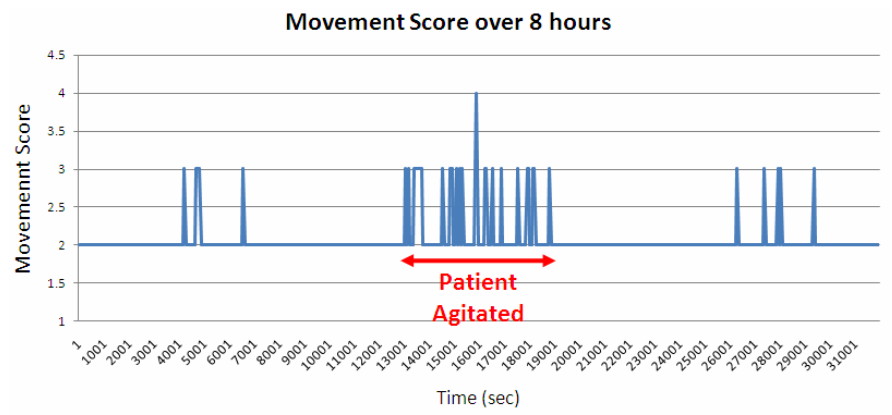

Figure 11. Movement Score gathered over 8 hours from a single patient
This stage in the work will be followed by a further trial involving a validation cohort of 60 patients where the possibility that correlations exist between currently employed scoring systems and Movement Score will be examined.

\section{CONCLUSION}

This paper has provided a system description and preliminary results for an ongoing clinical study currently being carried out at the Mid-Western Regional Hospital, Nenagh, Ireland. The system is designed to overcome a number of the logistical barriers to the adoption of wearable wireless technology in front-line medical care. These design attributes include custom packaging to adhere to hygiene standards and networking protocols to cope with difficult wireless propagation environments,

The goal of the trial is to determine if wireless inertial measurement technology can be employed to identify patients at risk of death or imminent clinical deterioration. The system measures cumulative movement and provides a score that will provide a robust early warning to clinical staff of clinical deterioration. In addition the system has been shown to be able to highlight events of interest such as patient agitation based on Movement Score.

\section{ACKNOWLEDGMENT}

The authors would like to thank the nursing staff of Nenagh Hospital for their assistance. The authors would like to acknowledge support by Science Foundation Ireland under grant 07/CE/I1147.

\section{REFERENCES}

[1] C. P. Subbe, M. Kruger, L. Gemmel, "Validation of a modified Early Warning Score in medical admissions", Quarterly Journal of Medicine, vol. 94, pp. 521-526, 2001

[2] J. Kellett, B. Deane, "The Simple Clinical Score predicts mortality for 30days after admission to an acute medical unit", Qual J Med, vol. 99, pp.771-81, 2006

[3] J. Kellett, B. Deane, M. Gleeson. "Derivation and validation of a score based on Hypotension, Oxygen saturation, low Temperature, ECG changes and Loss of independence (HOTEL) that predicts early mortality between 15 minutes and 24 hours after admission to an acute medical unit”. Resuscitation, vol. 78, pp. 52-58, 2008.

[4] M. Walsh, "A benchmark comparison between reconfigurable, intelligent and autonomous wireless inertial measurement and photonic technologies in rehabilitation", Accepted to 3rd European Conference on Technically Assisted Rehabilitation - TAR 2011.

[5] M. Hautefeuille, C. O'Mahony, B. O'Flynn, K. Khalil, F. Peters, “A MEMS-based WirelessMultisensor Module for Environmental Monitoring", Microelectronics Reliability, Vol. 48, pp. 906-910, 2008. 